\title{
PAK6: a potential anti-cancer target
}

\author{
Chan-Chan Gong ${ }^{1}$, Tong-Tong Li ${ }^{1}$, Dong-Sheng Pei ${ }^{\oplus 1,2, *}$ \\ ${ }^{1}$ Department of Pathology, Xuzhou Medical University, Xuzhou, China, 2Jiangsu Key Laboratory of Biological Cancer \\ Therapy, Xuzhou Medical University, Xuzhou, China
}

\begin{abstract}
p21-activated kinase 6 (PAK6) is a member of the PAK family of serine/threonine kinases that are known effectors of Rho GTPases Cdc42 and Rac. PAKs regulate a large number of complex cellular mechanisms, including cell motility, morphology, and tumor development. PAK6, initially cloned as an interacting partner of the androgen receptor (AR), is associated with an array of cellular processes implicated in tumor progression. However, the full biological implications of PAK6 activity during cancer remain poorly understood. In this review, we assess our current understanding of the physiological roles of classical PAK6 functionality in mammals, in addition to its emerging role in tumorigenesis.
\end{abstract}

Keywords: PAK6; androgen Receptor (AR); cancer therapy; miRNAs.

\section{INTRODUCTION}

PAKs were first discovered through the design of novel antifungal agents by Manser and colleagues (Manser et al., 1994). In the ensuing decades, PAKs biology has attracted significant attention. PAKs are regarded as oncogenes that stimulate cancer progression through a plethora of cell-based strategies, including promoting cell survival, proteolytic degradation of the extracellular matrix, cytoskeletal remodeling to create motor structures, the acceleration of angiogenesis, and the phosphorylation of effector substrates (Molli et al., 2009). Structurally, PAKs are characterized by their $\mathrm{N}$-terminal Cdc42/Rac interaction binding domain (CRIB) and conserved C-terminal kinase domain (Kumar et al., 2017). To date, six members of the PAK family have been discovered which have been divided into two classes: Group I (PAK1-3) and Group II (PAK4-6), based on the sequence and functional homology (Radu et al., 2013). Group II members lack obvious autoinhibitory domains which are present in Group I (Radu et al., 2013). PAK6 is less well characterized than the other members of Group II PAKs, and studies on PAK6 have mainly focused on its role as an interacting partner of AR in prostate cancer cells, shown to be independent of Rho GTPases (Schrantz et al., 2004). During recent years, it has been shown that PAK6

\footnotetext{
*Correspondence: D.-S. Pei. Department of Pathology, Xuzhou Medical University, 209 Tong-shan Road, Xuzhou, Jiangsu 221004, P.R.China. Tel: +86-0516-85582513. E-mail: dspei@xzhmu.edu.cn
}

is either deregulated or hyper-activated in a large number of human cancers (Kumar et al., 2017; Gao et al., 2013). Furthermore, PAK6 has been shown to be essential for many fundamental cellular processes typically deregulated in cancer, including cell migration, differentiation, and survival (Jaffer, Chernoff, 2002; Field, Manser, 2012). Given the aforementioned considerations, there is urgent need to decipher the mechanisms of PAK6 activation during human disease, particularly in the setting of cancer progression.

\section{STRUCTURE AND EXPRESSION OF PAK6}

PAK6 is located on chromosome $15 \mathrm{q} 15$ and is a 681 amino acid protein with a molecular mass of $75 \mathrm{kDa}$ (Yang et al., 2001). The PAK6 gene is $\sim 38 \mathrm{~kb}$ in length and comprises 16 exons, including 8 for 5'-UTRs, producing 17 transcripts (Lee et al., 2002). Like other members of the PAK family, PAK6 contains a conserved N-terminal $\mathrm{CRIB}$ and a $\mathrm{C}$-terminal kinase catalytic domain. The $\mathrm{N}$-terminus of PAK6 is similar to the CRIB domains of the previously characterized PAKs and contains six of the eight common CRIB domain residues (Wells, Jones, 2010; Hofmann, Shepelev, Chernoff, 2004; Ha et al., 2012). The C-terminal of PAK6 has more than $50 \%$ sequence similarity with PAK1-3, and high homology to PAK4 ( $80 \%$ homology), indicating alternative mechanisms of kinase regulation (Arias-Romero, Chernoff, 2008). PAK4, the most extensively studied Group II PAKs member, 
is highly expressed in the colon, testes and prostate of humans (Jaffer, Chernoff, 2002). PAK6 is highly expressed in the brain and testes, and expressed to low levels in prostate, breast, thyroid, kidney, and placenta. There is little detectable PAK6 expression in the ovaries, peripheral blood leukocytes, lymph nodes, uterus, spleen, intestine, liver, lung, thymus, or bone marrow (Yang et al., 2001).

\section{BIOLOGICAL FUNCTION OF PAK6}

PAK6 modulates a plethora of biological activities, including cell proliferation, apoptosis, invasion, metastasis and cytoskeletal rearrangements (Eswaran et al., 2007). As an exemplar, PAK6 overexpression has been shown to drive cell-cell dissemination (Fram et al., 2014). Fram and colleagues demonstrated that PAK6 binds to cell-cell adhesions, co-localizing with E-cadherin and IQ motif containing GTPase activating protein 1 (IQGAP1) at these regions (Fram et al., 2014). The interplay between the $\mathrm{C}$-terminal kinase catalytic domain of PAK 6 and the N-terminus of IQGAP1 plays a vital role in the formation of cell-cell junctions. The $\mathrm{N}$-terminus of PAK6 is necessary and sufficient for its targeting to cell-cell adhesions, suggesting its catalytic activity is not required (Morse et al., 2016). Additionally, PAK6 localization at cell-cell adhesions requires $\mathrm{Cdc} 42$, an evolutionarily conserved polarity protein that acts as an upstream activator of PAKs (Takahashi, Pryciak, 2007; Su et al., 2005; Baskaran et al., 2012). PAK6 promotes cell-cell dissociation through its ability to target cell-cell adhesion, as determined by epithelial cell colony escape assays, the key progress in epithelial mesenchymal transition (EMT) (Morse et al., 2016). Investigation of the molecular interactions leading to junctional dissociation and the identification of proteins involved in this process will be beneficial to our broader understanding of cancer progression.

PAK6 is highly expressed in brain tissue, particularly the cortical structure, suggesting a crucial role in the central nervous system (CNS) (Minden, 2012; Civiero et al., 2015). Although its precise function in the CNS remains unclear, several recent studies suggest a role for PAK6 during cognitive behavior. In learning and memory behavioral tests, PAK5/PAK6 double-knockout mice show slight learning and memory deficits and locomotor changes compared with wild-type controls (Nekrasova et al., 2008). Whilst PAK6 knockout mice were similar to wild type mice, PAK5 knockout mice displayed a modest loss in learning ability and memory function, which was less pronounced than the PAK5/PAK6 double knockout. This suggests at least a partial overlap between PAK6 and
PAK5 CNS functions. PAK5/PAK6 double-knockout mice also displayed alterations in neuronal morphological and synaptic contact arrangements that likely contribute to the defects. This highlights the interplay between Group II PAKs and their contribution to neuronal processes (Skoulakis et al., 2013).

A potential candidate that contributes to these processes is LIM kinase (LIMK), a substrate for several PAKs, including PAK6 (Cai et al., 2015). Defects in LIMK1 are associated with Williams syndrome, a neurodevelopmental disorder that causes learning and behavior disorders (Matsumoto, Kitani, Kalinec, 2011). In addition, PAK6 plays an essential role in weight gain unrelated to caloric intake and exercise (Furnari et al., 2014; Kreis, Barnier, 2009). PAK6 also decreases AR and estrogen receptor (ER) transcriptional expression. AR participates in body weight determination and challenge, providing further evidence of a role for PAK6 during weight gain, independent of exercise and calorie intake (Lee et al., 2002; Narayanan, Coss, Dalton, 2017). More importantly, PAK6 also participates in CNS pathophysiology following traumatic brain injury (TBI), the dominant cause of disability and death globally. TBI leads to secondary tissue loss and glial scar formation and impairs the regeneration associated with functional disabilities (Zhao et al., 2011). Likewise, PAK6 expression is markedly increased in astrocytes and neurons and is associated with astrocyte proliferation following spinal cord injury (SCI) in adult rats (Chen, Zhao, Shen, 2011). The known activation of PAK6 by the MAP kinase kinase 6 (MKK6)/p38 pathway further implicates its involvement in stress-related signaling (Kaur et al., 2004). Furthermore, proliferating cell nuclear antigen (PCNA) is a cyclin involved in mitotic G1/S progression and that is up-regulated in astrocytes after SCI (Chen, Zhao, Shen, 2011). PAK6 induction in astrocytes accompanies this upregulation (Chen, Zhao, Shen, 2011). Further studies investigating the function of PAK6 during CNS injury and repair may reveal novel molecular pathways that can be manipulated during the treatment of CNS trauma.

\section{THE ROLE OF PAK6 IN CANCER}

Cancer is a progressive disease caused by genomic instability, resulting in chromosome translocations (Hofmann, Shepelev, Chernoff, 2004). Considering the highly unpredictable and complex natural history of cancer, it is of the utmost important to explore molecular pathways, identify prognostic factors, and develop more sensitive and accurate integrated prognostic models (Torre et al., 2015). Aberrant expression of PAK6 is 
observed in several cancers, with its contribution to prostate cancer development and progression following androgen deprivation therapy well defined.

\section{Prostate cancer}

Prostate cancer is one of the most common causes of cancer deaths and represents one of the most dominant cancers in males in the United States and Western Europe, largely due to the limitations of the current treatment modalities, particularly following metastasis (Liu et al., $2013 b$ ). The AR is a member of the steroid hormone receptor of the larger nuclear receptor family and plays an essential role in the development of prostate cancer (Narayanan, Coss, Dalton, 2017). Tong and colleagues revealed that PAK6 could inhibit prostate cancer growth in vivo by promoting AR ubiquitin-mediated degradation through AR and Mdm2 phosphorylation (Liu et al., 2013a). This result is surprising since PAK6 is highly expressed in metastatic and primary cancers. In prostate cancer cell lines, PAK6 expression is significantly higher in malignant prostate tissue and correlates with tumor grade (Liu et al., 2013b). Similarly, increased PAK6 staining significantly correlates with a higher Gleason score (Kaur et al., 2008). One interpretation of these findings is that the expression of PAK6 increases to inhibit excessive AR expression and tumor aggressiveness (Liu et al., 2013a). Indeed, increased nuclear translocation of AR correlates with high malignancy in prostate cancer (Liu et al., 2013a). The stress-induced activation of PAK6 in response to enhanced AR activity may explain its overexpression in prostate cancer. PAK6 may further influence carcinogenesis and prostate cancer progression through diverse signal transduction pathways including protein phosphatase 1B (PP1B) and IQGAP1, both of which are PAK6 interacting partners (Kaur et al., 2008; Fram et al., 2014). IQGAP1 plays a critical role in cell adhesion and coordinates multiple signaling pathways in many cell types (Choi, Anderson, 2016; Abel et al., 2015).

Various microRNAs (miRs) are involved in prostate cancer development and progression (Farazi et al., 2013). Previous studies have demonstrated that PAK6 is directly targeted by miR-328 (Liu et al., 2015a) and a miR-23aPAK6-LIMK1 regulatory pathway has been shown to promote prostate cancer metastasis (Cai et al., 2015). PAK6 is negatively correlated to miR-23a in prostate cancer tissue (Cai et al., 2015). MiR-23a reduces PAK6 expression by directly binding to complementary sites in the 3'-UTR of PAK6 (Cai et al., 2015). The expression of miR-23a suppresses LIMK and cofilin phosphorylation, which in turn inhibits the formation of actin filaments and stress fibers required for cell invasion and motility (Cai et al., 2015). PAK6 therefore represents a valuable therapeutic target for prostate cancer treatment.

PAK6 is implicated in mediating the response to radiotherapy and chemotherapy during prostate cancer treatment (Zhang et al., 2010). PAK6 silencing enhances the chemosensitivity of docetaxel, but the molecular basis for this remains unclear. Min and coworkers found that PAK6 mediates the resistance of prostate cancer cells to radiation therapy (Zhang et al., 2010), with PAK6 inhibition decreasing the levels of radiation-induced apoptosis in prostate cancer cells (Wen et al., 2009). It is well-known that local and systemic recurrence after radiotherapy (a curative treatment for clinically localized prostate cancer) is high, suggesting that drug resistance is a common event. The underlying mechanisms by which PAK6 mediates radiation therapy responses involves changes in cell cycle distribution, altered BAD phosphorylation, and impaired DNA double-strand break repair (Zhang et al., 2010). In addition, PAK6 expression is significantly associated with Proteasome beta-4 subunit (PSMB4) (Zapatero et al., 2014). The over-expression of PSMB4 and its role in proteasome mediated survival inhibition has been demonstrated in several metastatic and primary solid tumors (Liu et al., 2016). Taken together, these studies highlight PAK6 as a viable target to improve the effectiveness of radiotherapy during prostate cancer treatment.

\section{Hepatocellular carcinoma}

Although a variety of prognostic indicators of hepatocellular carcinoma (HCC) (including osteopontin, transforming growth factor and vascular endothelial growth factor) have originated from a large number of clinical and basic research efforts, effective treatment modalities remain rare, and liver transplantation is the primary therapeutic strategy for early HCC patients (Chen et al., 2014). New strategies to improve HCC treatment are urgently required. PAK6 expression decreases during $\mathrm{HCC}$ development with its inhibition associated with a poor clinical outcome (Liu et al., 2015b). Furthermore, under the epigenetic regulation of multiple inhibitory complex 2 (PRC2), PAK6 acts as a tumor suppressor during HCC development, which is dependent on its nuclear translocation and kinase activity (Liu et al., 2015b). PRC2 has both oncogenic and tumor suppressor functions and catalyzes the trimethylation of histone H3 lysine 27 (H3K27), an inhibitory chromatin marker that correlates with gene silencing (Holoch, Margueron, 2017). EZH2, the catalytic subunit of 
PRC2, is negatively associated with PAK6 in HCC, providing further evidence of its tumor suppressive role during hepatocarcinogenesis (Jain, Di Croce, 2016; Liu et al., 2015b). These findings demonstrate that PAK6 suppression contributes to HCC (Figure 1). In contrast, retrospective studies suggest that PAK6 is markedly elevated in HCC specimens and positively associated with HCC cell proliferation (Chen et al., 2014). High expression of PAK6 also correlates with the Edmondsone Steiner classification, poor prognosis and the number of tumor nodules (Chen et al., 2014). However, the detailed molecular mechanisms of how PAK6 over-expression contributes to $\mathrm{HCC}$ remains poorly understood. Given the retrospective nature of the study and the limitations of investigating patients in a single institution of a single histological type, these conclusions require validation in large-scale, multi-agency and prospective studies. Further studies into the relationship of PAK6 and PRC2 may fully reveal its role during HCC development. Interestingly, the contradictory findings regarding the role of PAK6 in cancer have similarly been reported in prostate cancer, and may thus be a consequence of the intricate molecular functions of PAK6. DNA methylation analysis has also demonstrated hypermethylation in the PAK6 promoter in prostate cancer cells, which is associated with gene transcriptional silencing. These genes are connected to the inhibition of tumor progression and are known tumor suppressors (Lee et al., 2010). It will be interesting to determine whether PAK6 has both oncogenic and tumor suppressive functions during the progression and development of cancer in future studies.

\section{Colon cancer}

Colon cancer (CC) that is located in the sigmoid or descending colon is defined as left colorectal cancer (LCC), whilst that located in the transverse colon, ascending colon, and cecum is defined as right colorectal cancer (RCC) (Yang et al., 2017). CC is one of the leading causes of cancer related mortality worldwide, the median survival rate for patients remaining poor (Feng et al., 2017). Although therapeutics targeting cell signaling pathways remains a pillar of current cancer research, drug resistance remains a major obstacle (Chen, Lin, Chen, 2017). Systemic adjuvant chemotherapy based on 5-fluorouracil (5-FU) has been proposed for patients with a high recurrence risk and has been shown to be beneficial in many trials (Mori et al., 2017). Chen and colleagues demonstrated that PAK6 reduces the sensitivity of 5-FU drugs and the combination of PAK6 inhibition and 5-FU treatment results in a significant reduction in colon cancer cell survival (Chen et al., 2015). This suggests that PAK6 expression may provide prognostic value in determining optimal individualized treatment regimens during colon cancer diagnosis, by differentiating those patients predicted to respond to postoperative chemotherapy. Most importantly, PAK6 expression is upregulated in colon cancer tissue compared with normal colon epithelial cells (Tian et al., 2015). Studies have revealed that PAK6 signaling is linked to miR-429-mediated colon cancer cell invasion and migration (Tian et al., 2015). miR-429 deregulation is implicated in pancreatic, oropharyngeal carcinoma, colon cancer and non-small cell lung cancer (NSCLC) (Wang et al., 2016). PAK6 expression is negatively regulated by miR-429 in colon cancer cells (Tian et al., 2015).

It is therefore clear that PAK6 represents an independent prognostic factor for colon cancer patients. The expansion and molecular mechanisms of PAK6 specific substrates will further our understanding of the role of PAK6 during tumorigenesis.

\section{Other cancers}

In contrast to PAK4, PAK6 function during tumor progression remains poorly characterized. However, PAK6 has been suggested to play a crucial role in the progression and development of lung cancer, gastric cancer (GC) and clear cell renal cell carcinoma (ccRCC) (Table I).

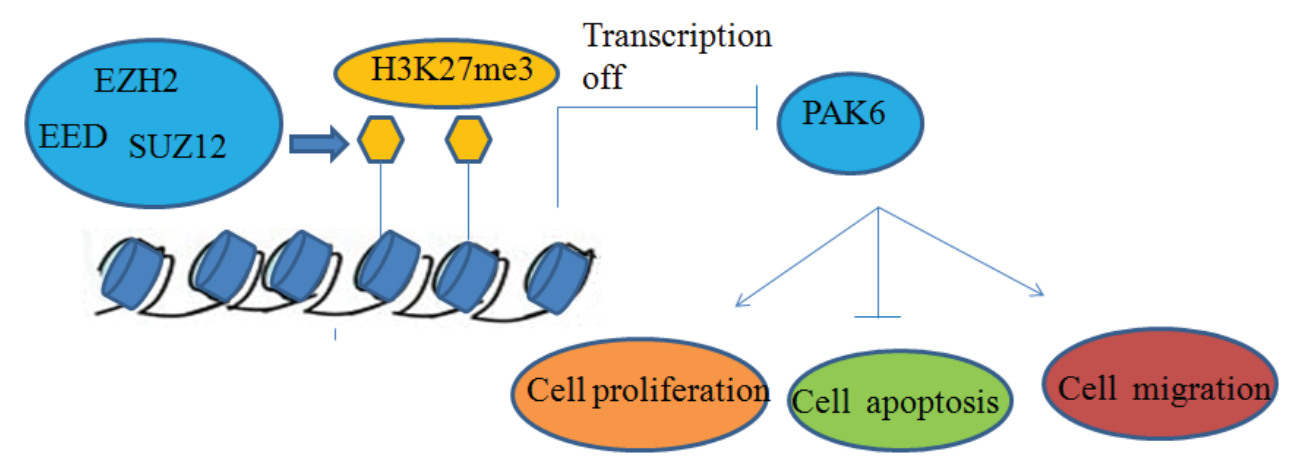

FIGURE 1 - The epigenetic regulation of PAK6 in HCC. 
Low levels of PAK6 have been documented to correlate with poor overall survival and recurrence-free survival in ccRCC patients following nephrectomy (Liu et al., 2014). These findings suggest that PAK6 in ccRCC acts as a tumor suppressor gene and that its low expression in surgical tumor tissue predicts a poorer clinical outcome. This suggests that patients with early stage ccRCC should be treated under strict surveillance (Liu et al., 2014). PAK1 is overexpressed in RCC tissue and may represent a potential therapeutic target (O'Sullivan et al., 2007). In addition, compared to surrounding non-tumor mucosa, PAK6 expression is increased in GC tissue and correlates with poor prognosis following chemotherapy (Jiang et al., 2017). Since chemotherapy is recommended as a standard postoperative treatment for advanced $\mathrm{GC}$, individualizing patient management based on PAK6 expression may be beneficial (Kawai et al., 2017). Epigenetic changes associated with the histological features of each cancer may aid our understanding of the molecular context of clinical pathological diversity in human cancers. In addition, since PAK6 inhibition decreases cell proliferation, invasion and motility of cigarette smoke treated cells (Raja et al., 2016), studies investigating PAK6 as a potential therapeutic target for NSCLC, particularly in smokers, should be prioritized (Raja et al., 2016). Understanding the complexity of cancer relies on clarifying the basic regulatory network of intercellular and cellular levels and their temporal dimensions. Significantly, it has been reported that PAK6 methylation acts as a marker of adenocarcinoma (ACA) allowing its distinction from squamous cell carcinoma (SCC) (Lee et al., 2010). Changes in DNA methylation remain one of the most common molecular alterations in human neoplasia which may represent an early event occurring during cancer development, providing a unique perspective as an early detection marker (Laird, 2003). Exploration of the detailed molecular mechanisms of PAK6 in various tumors and its potential clinical value is therefore highly encouraged.

\section{CONCLUSIONS}

Although we remain at the early stages of understanding the fundamental roles of Group II PAKs, there is evidence that the two subgroups vary in terms of their substrate preferences, biochemical properties, and cellular and developmental functions (Dammann,

TABLE I - Aberrant expression of PAK6 in tumors

\begin{tabular}{|c|c|c|c|c|}
\hline Tumor & Mechnism & Potential target & Effect & References \\
\hline \multirow[t]{3}{*}{ Prostate cancer } & $\begin{array}{l}\text { AR-ubiquitin-mediated } \\
\text { degradation through E3 } \\
\text { ligase Mdm2 }\end{array}$ & AR & $\begin{array}{l}\text { Aberrent expressed in prostate } \\
\text { cancer }\end{array}$ & (Liu et al., 2013a) \\
\hline & miR-23a-PAK6-LIMK1 & miR-23a/LIMK1 & & (Cai et al., 2015) \\
\hline & miR-328-PAK6 & $\operatorname{miR}-328$ & & (Liu et al., 2015a) \\
\hline Lung cancer (NSCLC) & & & $\begin{array}{l}\text { Promotion of cell } \\
\text { proliferation, migration } \\
\text { and invasion }\end{array}$ & (Raja et al., 2016) \\
\hline $\mathrm{HCC}$ & & & Aberrently expressed in $\mathrm{HCC}$ & $\begin{array}{l}\text { (Chen et al., 2014; } \\
\text { Liu et al., 2015b) }\end{array}$ \\
\hline Breast cancer & & & $\begin{array}{l}\text { Over-expressed in breast } \\
\text { cancer cells }\end{array}$ & (Kaur et al., 2008) \\
\hline Gastric cancer & & & $\begin{array}{l}\text { Over-expressed in gastric } \\
\text { cancer }\end{array}$ & (Jiang et al., 2017) \\
\hline Thyroid cancer & & & $\begin{array}{l}\text { Expressed in normal and } \\
\text { malignant thyroid tissues }\end{array}$ & (McCarty et al., 2010) \\
\hline
\end{tabular}

NSCLC: non-small cell lung cancer AKT: protein kinase B HCC: hepatocellular carcinoma OS: overall survival RFS: recurrencefree survival. 


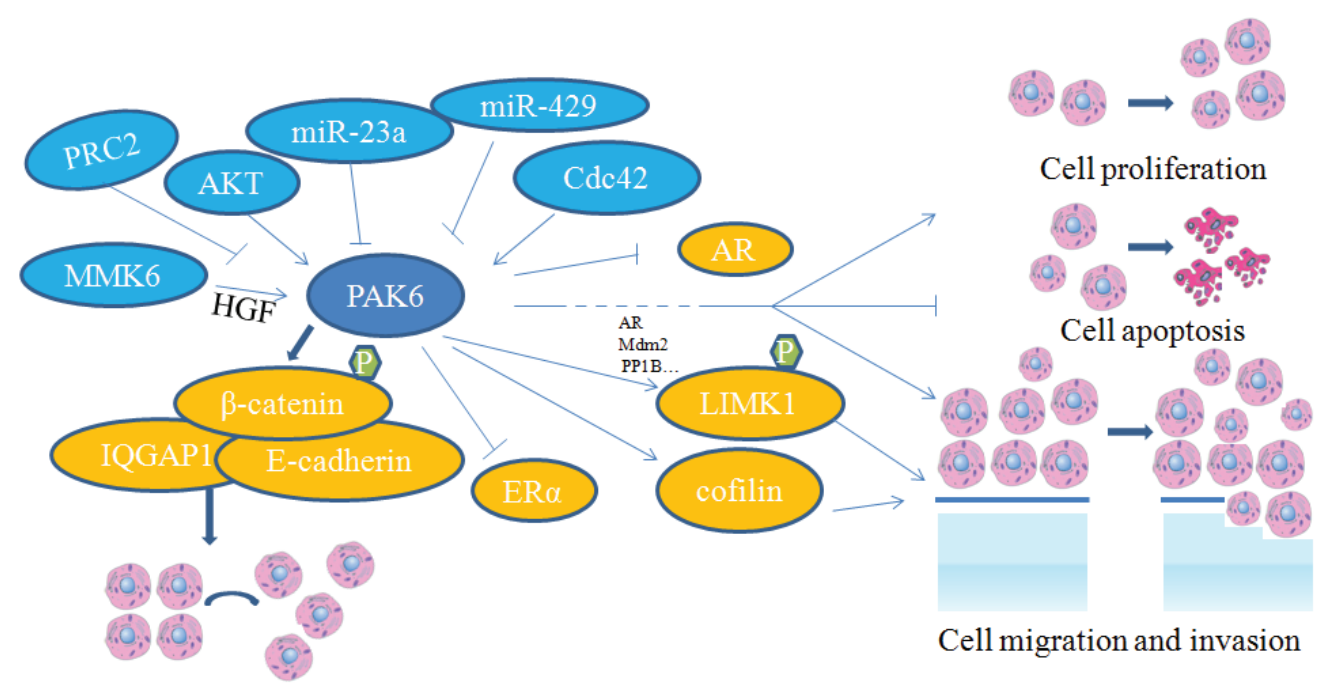

Cell-cell dissociation

FIGURE 2 - The signal transduction cascade of PAK6 in cancer.

Khare, Gasche, 2014). Accumulating data also shows that PAK6 is aberrantly expressed in a large number of human diseases, including cancer (Rudolph et al., 2014). With regards to the function of PAK6 in cancer, we have a reasonable understanding of the signal-framework (Figure 2), but some basic problems remain unanswered. Whilst most PAKs are considered oncogenes, emerging evidence suggests that different PAKs have different or even opposite functions during cancer development. These differences may be caused by varying levels of regulation, including optimal phosphorylation sites, different effects of Cdc42 interactions, and alternative substrates, including AR for PAK6. Unravelling the answers to these questions will improve our understanding of these new kinases, and the signaling pathways through which they mediate their ultimate cellular functions. Further studies regarding the function of PAK6 are now warranted in a range of contexts to further explore its role in tumor development. PAK6 may emerge as one of most frequently up-regulated pathways in human cancer, revealing members of the PAK family as novel anti-cancer therapeutic targets.

\section{ACKNOWLEDGMENTS}

This work was supported by the National Natural Science Foundation of China (No. 81572349), Jiangsu Provincial Medical Talent (ZDRCA2016055) and the Science and Technology Department of Jiangsu Province (BK20141149).

\section{CONFLICTS OF INTEREST}

\section{REFERENCES}

Abel AM, Schuldt KM, Rajasekaran K, Hwang D, Riese MJ, Rao S, et al. IQGAP1: insights into the function of a molecular puppeteer. Mol Immunol. 2015;65(2):336-49.

Arias-Romero LE, Chernoff J. A tale of two Paks. Biol Cell. 2008;100(2):97-108.

Baskaran Y, Ng YW, Selamat W, Ling FT, Manser E. Group I and II mammalian PAKs have different modes of activation by Cdc42. EMBO Rep. 2012;13(7):653-9.

Cai S, Chen R, Li X, Cai Y, Ye Z, Li S, et al. Downregulation of microRNA-23a suppresses prostate cancer metastasis by targeting the PAK6-LIMK1 signaling pathway. Oncotarget. 2015;6(6):3904-17.

Chen CC, Lin JC, Chen KW. Comparison cisplatin with cisplatin plus $5 \mathrm{FU}$ in head and neck cancer patients received postoperative chemoradiotherapy. Oral Oncol. 2017;69:11-14.

Chen H, Miao J, Li H, Wang C, Li J, Zhu Y, et al. Expression and prognostic significance of $\mathrm{p} 21$-activated kinase 6 in hepatocellular carcinoma. J Surg Res. 2014;189(1):81-8.

Chen J, Lu H, Yan D, Cui F, Wang X, Yu F, et al. PAK6 increase chemoresistance and is a prognostic marker for stage II and III colon cancer patients undergoing 5-FU based chemotherapy. Oncotarget. 2015;6(1):355-67.

All authors declare no conflicts of interest. 
Chen XD, Zhao W, Shen AG. Expression and role of PAK6 after spinal cord injury in adult rat. Chin J Traumatol. 2011;14(5):27781.

Choi S, Anderson RA. IQGAP1 is a phosphoinositide effector and kinase scaffold. Adv Biol Regul. 2016; 60:29-35.

Civiero L, Cirnaru MD, Beilina A, Rodella U, Russo I, Belluzzi E, et al. Leucine-rich repeat kinase 2 interacts with p21-activated kinase 6 to control neurite complexity in mammalian brain. $\mathrm{J}$ Neurochem. 2015;135(6):1242-56.

Dammann K, Khare V, Gasche C. Tracing PAKs from GI inflammation to cancer. Gut. 2014;63(7):1173-84.

Eswaran J, Lee WH, Debreczeni JE, Filippakopoulos P, Turnbull A, Fedorov O, et al. Crystal Structures of the p21-activated kinases PAK4, PAK5, and PAK6 reveal catalytic domain plasticity of active group II PAKs. Structure. 2007;15(2):201-13.

Farazi TA, Hoell JI, Morozov P, Tuschl T. MicroRNAs in human cancer. Adv Exp Med Biol. 2013;774:1-20.

Feng H, Liu Y, Bian X, Zhou F, Liu Y. ALDH1A3 affects colon cancer in vitro proliferation and invasion depending on CXCR4 status. Br J Cancer. 2018;118(2):224-232.

Field J, Manser E. The PAKs come of age: Celebrating 18 years of discovery. Cell Logist. 2012;2(2):54-58.

Fram S, King H, Sacks DB, Wells CM. A PAK6-IQGAP1 complex promotes disassembly of cell-cell adhesions. Cell Mol Life Sci. 2014;71(14):2759-73.

Furnari MA, Jobes ML, Nekrasova T, Minden A, Wagner GC. Differential sensitivity of Pak5, Pak6, and Pak5/Pak6 doubleknockout mice to the stimulant effects of amphetamine and exercise-induced alterations in body weight. Nutr Neurosci. 2014;17(3):109-15.

Gao J, Ha BH, Lou HJ, Morse EM, Zhang R, Calderwood DA, et al. Substrate and inhibitor specificity of the type II p21activated kinase, PAK6. PLoS One. 2013;8(10):e77818.

Ha BH, Davis MJ, Chen C, Lou HJ, Gao J, Zhang R, et al. Type II p21-activated kinases (PAKs) are regulated by an autoinhibitory pseudosubstrate. Proc Natl Acad Sci USA. 2012;109(40):16107-12.

Hofmann C, Shepelev M, Chernoff J. The genetics of Pak. J Cell Sci. 2004;117(19):4343-4354.
Holoch D, Margueron R. Mechanisms regulating PRC2 recruitment and enzymatic activity. Trends Biochem Sci. 2017;42(7):531-542.

Jaffer ZM, Chernoff J. p21-activated kinases: three more join the Pak. Int J Biochem Cell Biol. 2002;34(7):713-7.

Jain P, Di Croce L. Mutations and deletions of PRC2 in prostate cancer. Bioessays. 2016;38(5):446-54.

Jiang Y, Liu W, Li T, Hu Y, Chen S, Xi S, et al. Prognostic and predictive value of p21-activated kinase 6 Associated support vector machine classifier in gastric cancer treated by 5 -fluorouracil/oxaliplatin chemotherapy. EBioMedicine. 2017;22:78-88.

Kaur R, Liu X, Gjoerup O, Zhang A, Yuan X, Balk SP, et al. Activation of p21-activated kinase 6 by MAP kinase kinase 6 and p38 MAP kinase. J Biol Chem. 2004;280(5):3323-3330.

Kaur R, Yuan X, Lu ML, Balk SP. Increased PAK6 expression in prostate cancer and identification of PAK6 associated proteins. Prostate. 2008;68(14):1510-6.

Kawai A, Matsumoto H, Endou Y, Honda Y, Kubota H, Higashida M, et al. Repeated combined chemotherapy with cisplatin lowers carnitine levels in gastric cancer patients. Ann Nutr Metab. 2017;71(3-4):261-265.

Kreis P, Barnier JV. PAK signalling in neuronal physiology. Cell Signal. 2009;21(3):384-93.

Kumar R, Sanawar R, Li X, Li F. Structure, biochemistry, and biology of PAK kinases. Gene. 2017;605:20-31.

Laird PW. The power and the promise of DNA methylation markers. Nat Rev Cancer. 2003;3(4):253-66.

Lee EJ, McClelland M, Wang Y, Long F, Choi SH, Lee JH. Distinct DNA methylation profiles between adenocarcinoma and squamous cell carcinoma of human uterine cervix. Oncol Res. 2010;18(9):401-8.

Lee SR, Ramos SM, Ko A, Masiello D, Swanson KD, Lu $\mathrm{ML}$, et al. AR and ER interaction with a p21-activated kinase (PAK6). Mol Endocrinol. 2002;16(1):85-99.

Liu C, Zhang L, Huang Y, Lu K, Tao T, Chen S, et al. MicroRNA328 directly targets p21activated protein kinase 6 inhibiting prostate cancer proliferation and enhancing docetaxel sensitivity. Mol Med Rep. 2015a;12(5):7389-95. 
Liu R, Lu S, Deng Y, Yang S, He S, Cai J, et al. PSMB4 expression associates with epithelial ovarian cancer growth and poor prognosis. Arch Gynecol Obstet. 2016;293(6):1297-307.

Liu T, Li Y, Gu H, Zhu G, Li J, Cao L, et al. p21-Activated kinase 6 (PAK6) inhibits prostate cancer growth via phosphorylation of androgen receptor and tumorigenic E3 ligase murine double minute-2 (Mdm2). J Biol Chem. 2013a;288(5):3359-69.

Liu W, Liu H, Liu Y, Xu L, Zhang W, Zhu Y, et al. Prognostic significance of $\mathrm{p} 21$-activated kinase 6 expression in patients with clear cell renal cell carcinoma. Ann Surg Oncol. 2014;21(Suppl 4):S575-83.

Liu W, Liu Y, Liu H, Zhang W, Fu Q, Xu J, et al. Tumor suppressive function of $\mathrm{p} 21$-activated kinase 6 in hepatocellular carcinoma. J Biol Chem. 2015b;290(47):28489-501.

Liu X, Busby J, John C, Wei J, Yuan X, Lu ML. Direct interaction between AR and PAK6 in androgen-stimulated PAK6 activation. PLoS One. 2013b;8(10): e77367.

Manser E, Leung T, Salihuddin H, Zhao ZS, Lim L. A brain serine/threonine protein kinase activated by $\mathrm{Cdc} 42$ and Rac1. Nature. 1994;367(6458):40-6.

Matsumoto N, Kitani R, Kalinec F. Linking LIMK1 deficiency to hyperacusis and progressive hearing loss in individuals with Williams syndrome. Commun Integr Biol. 2011;4(2):208-10.

McCarty SK, Saji M, Zhang X, Jarjoura D, Fusco A, Vasko VV, et al. Group I p21-activated kinases regulate thyroid cancer cell migration and are overexpressed and activated in thyroid cancer invasion. Endocr Relat Cancer. 2010;17(4):989-99.

Minden A. PAK4-6 in cancer and neuronal development. Cell Logist. 2012;2(2):95-104.

Molli PR, Li DQ, Murray BW, Rayala SK, Kumar R. PAK signaling in oncogenesis. Oncogene. 2009;28(28):2545-55.

Mori R, M Futamura, T Tanahashi, Y Tanaka, N Matsuhashi, $\mathrm{K}$ Yamaguchi, et al. 5FU resistance caused by reduced fluoro-deoxyuridine monophosphate and its reversal using deoxyuridine. Oncol Lett. 2017;14(3):3162-3168.

Morse EM, Sun X, Olberding JR, Ha BH, Boggon TJ, Calderwood DA. PAK6 targets to cell-cell adhesions through its $\mathrm{N}$-terminus in a Cdc42-dependent manner to drive epithelial colony escape. J Cell Sci. 2016;129(2):380-93.
Narayanan R, Coss CC, Dalton JT. Development of selective androgen receptor modulators (SARMs). Mol Cell Endocrinol. 2017;15(17):30340-4.

Nekrasova T, Jobes ML, Ting JH, Wagner GC, Minden A. Targeted disruption of the Pak5 and Pak6 genes in mice leads to deficits in learning and locomotion. Dev Biol. 2008;322(1):95108.

O’Sullivan GC, Tangney M, Casey G, Ambrose M, Houston A, Barry OP. Modulation of p21-activated kinase 1 alters the behavior of renal cell carcinoma. Int J Cancer. 2007;121(9):193040 .

Radu M, Semenova G, Kosoff R, Chernoff J. PAK signalling during the development and progression of cancer. Nature Reviews Cancer. 2013;14(1):13-25.

Raja R, Sahasrabuddhe NA, Radhakrishnan A, Syed N, Solanki HS, Puttamallesh VN, et al. Chronic exposure to cigarette smoke leads to activation of p21 (RAC1)-activated kinase 6 (PAK6) in non-small cell lung cancer cells. Oncotarget. 2016;7(38):6122961245.

Rudolph J, Crawford JJ, Hoeflich KP, Wang W. Inhibitors of p21-Activated Kinases (PAKs). J Med Chem. 2014;58(1):111129.

Schrantz N, da Silva Correia J, Fowler B, Ge Q, Sun Z, Bokoch GM. Mechanism of p21-activated kinase 6-mediated inhibition of androgen receptor signaling. J Biol Chem. 2004;279(3):192231.

Skoulakis EMC, Furnari MA, Jobes ML, Nekrasova T, Minden A, Wagner GC. Functional Deficits in Pak5, Pak6 and Pak5/Pak6 Knockout Mice. PLoS ONE. 2013;8(4):e61321.

Su Z, Osborne MJ, Xu P, Xu X, Li Y, Ni F. A bivalent dissectional analysis of the high-affinity interactions between $\mathrm{Cdc} 42$ and the Cdc42/Rac interactive binding domains of signaling kinases in Candida albicans. Biochemistry. 2005;44(50):16461-74.

Takahashi S, Pryciak PM. Identification of novel membranebinding domains in multiple yeast $\mathrm{Cdc} 42$ effectors. Mol Biol Cell. 2007;18(12):4945-56.

Tian X, Wei Z, Wang J, Liu P, Qin Y, Zhong M. MicroRNA-429 inhibits the migration and invasion of colon cancer cells by targeting PAK6/cofilin signaling. Oncol Rep. 2015;34(2):70714. 
Torre LA, Bray F, Siegel RL, Ferlay J, Lortet-Tieulent J, Jemal A. Global cancer statistics, 2012. CA Cancer J Clin. 2015;65(2):87-108.

Wang F, Jiang C, Sun Q, Yan F, Wang L, Fu Z, et al. Downregulation of miR429 and inhibition of cell migration and invasion in nasopharyngeal carcinoma. Mol Med Rep. 2016;13(4):3236-42.

Wells CM, Jones GE. The emerging importance of group II PAKs. Biochem J. 2010;425(3):465-73.

Wen X, Li X, Liao B, Liu Y, Wu J, Yuan X, et al. Knockdown of p21-activated kinase 6 inhibits prostate cancer growth and enhances chemosensitivity to docetaxel. Urology. 2009;73(6):1407-11.

Yang F, Li X, Sharma M, Zarnegar M, Lim B, Sun Z. Androgen receptor specifically interacts with a novel p21-activated kinase, PAK6. J Biol Chem. 2001;276(18):15345-53.
Yang L, He W, Yang Q, Kong P, Xie Q, Jiang C, et al. Combination of primary tumor location and mismatch repair status guides adjuvant chemotherapy in stage II colon cancer. Oncotarget. 2017;8(58):99136-99149.

Zapatero A, Morente M, Nieto S, Martin de Vidales C, Lopez C, Adrados M, et al. Predictive value of PAK6 and PSMB4 expression in patients with localized prostate cancer treated with dose-escalation radiation therapy and androgen deprivation therapy. Urol Oncol. 2014;32(8):1327-32.

Zhang M, Siedow M, Saia G, Chakravarti A. Inhibition of p21activated kinase 6 (PAK6) increases radiosensitivity of prostate cancer cells. Prostate. 2010;70(8):807-16.

Zhao W, Yang J, Shi W, Wu X, Shao B, Wu Q, et al. Upregulation of 21 -activated Kinase 6 in rat brain cortex after traumatic brain injury. J Mol Histol. 2011;42(3):195-203.

Received for publication on $10^{\text {th }}$ July 2018 Accepted for publication on $09^{\text {th }}$ August 2018 